\title{
Analysis of widely targeted metabolites of the euhalophyte Suaeda salsa under saline conditions provides new insights into salt tolerance and nutritional value in halophytic species
}

\author{
Qiang Li and Jie Song ${ }^{*}$ (D)
}

\begin{abstract}
Background: Suaeda salsa L. (S. salsa) is an annual euhalophyte with high salt tolerance and high value as an oil crop, traditional Chinese medicine and vegetable. However, there are few comprehensive studies on the metabolomics of $S$. salsa under saline conditions.

Results: Seedlings of S. salsa were cultured with 0,200 and $500 \mathrm{mM} \mathrm{NaCl}$ for two days. Then, widely targeted metabolites were detected with ultra performance liquid chromatography and tandem mass spectrometry. A total of 639 metabolites were annotated. Among these, 253 metabolites were differential metabolites. Salt treatment increased the content of certain metabolites, such as nucleotide and its derivates, organic acids, the content of amino acids, lipids such as alinolenic acid, and certain antioxidants such as quercetin. These substances may be correlated to osmotic tolerance, increased antioxidant activity, and medical and nutritional value in the species.

Conclusion: This study comprehensively analyzed the metabolic response of $S$. salsa under salinity from the perspective of omics, and provides an important theoretical basis for understanding salt tolerance and evaluating nutritional value in the species.
\end{abstract}

Keywords: Antioxidant activity, Halophyte, Salinity, Suaeda salsa, Widely targeted metabolites

\section{Background}

Soil salinization has seriously affected agricultural production and food security. More than 800 million hectares of land worldwide are affected by soil salinization, which accounts for approximately $6 \%$ of the world's total land area, and this would result in reduced food production [1]. Meanwhile, the increasing amount of arable land lost to urban sprawl is forcing agricultural production into marginal areas [2]. Halophytes can be used as food, medicine and feed, and for restoring salinization and land contaminated with heavy metals.

\footnotetext{
* Correspondence: songjieever@163.com

Shandong Provincial Key Laboratory of Plant Stress, College of Life Science, Shandong Normal University, 88 Wenhua East Road, Jinan 250014, People's
} Republic of China

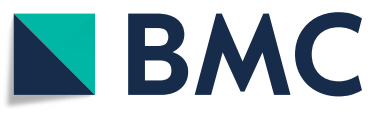

C The Author(s). 2019 Open Access This article is distributed under the terms of the Creative Commons Attribution 4.0 International License (http://creativecommons.org/licenses/by/4.0/), which permits unrestricted use, distribution, and reproduction in any medium, provided you give appropriate credit to the original author(s) and the source, provide a link to the Creative Commons license, and indicate if changes were made. The Creative Commons Public Domain Dedication waiver (http://creativecommons.org/publicdomain/zero/1.0/) applies to the data made available in this article, unless otherwise stated. phenotype of an organism [3, 4]. The total number of metabolites in plants is approximately 200,000 [5, 6]. Changes in the type and amount of metabolites can show how the organism adapts to environmental changes [7, 8]. Metabolomics is a method of qualitatively and quantitatively analyzing all metabolites in an organism $[6,9]$. Plant metabolism was disturbed under abiotic stress, and plants need to regulate metabolic levels to maintain basic metabolism and reach new homeostasis [10]. Hence, metabolomics was the most direct tool for studying this process $[11,12]$. During this process, the change in primary metabolism was most pronounced and also showed the general trend of plant response to abiotic stress. It involved the accumulation of compatible solutes such as sugars and sugar alcohols, amino acids, 
etc. to cope with osmotic stress [13]. However, changed in secondary metabolism were more specific for different species and stress conditions, such as modification and interaction of enzyme proteins [14], increase in asparagine [15], accumulation of flavonoids and other phenols [16], to scavenge ROS and act as a signal regulator substance [13]. At present, metabolomics has been playing an important role in the analysis of regional differences in wild rice [17], plant tolerance to abiotic stress [10], nitrogen metabolism [18], and the phenotypic variation analysis of Saccharomyces cerevisiae [19]. For example, under salt stress, co-induced glycolysis and sucrose metabolism and the co-reduction of the methylation cycle occur in Arabidopsis under salt stress [20]. Furthermore, small molecular organic solutes involved in the resistance to osmotic stress in Zea mays are significantly induced at high salinity, and this is found to be stronger in the shoots, when compared to the roots [21]. In salttolerant Hordeum vulgare L., the levels of hexose phosphate, TCA circulating intermediates, and metabolites involved in cell protection increases with the increase in salt concentration [22]. These studies have found pathways and metabolites that play important roles in salt tolerance. Therefore, metabolomics provides an important basis for salt response studies in plants. Suaeda salsa ( $S$. salsa) has stronger salt tolerance, and its metabolic level changes under salt stress. Hence, it has high value for research.

S. salsa is an euhalophyte with high salt tolerance during germination [23-26], vegetative growth [27] and reproductive stages [28-31]. The species is considered to have potential as a vegetable and oilseed crop [32, 33], and it is rich in protein, crude fiber, carotenoids and amino acids [34]. Furthermore, the species has high value as a medicine, and is a promising model for understanding salt tolerance [34]. The content of flavanols in S. salsa is much higher in July $\left(98.8 \mathrm{mg} \mathrm{g}^{-1} \mathrm{DW}\right)$ than in the other months, and the extracts in July have the highest antioxidant activity in vitro [34]. S. salsa has high ability to maintain ion homeostasis. For example, SsNHX1 and SsSOS1 are involved in maintaining $\mathrm{Na}^{+}$ homeostasis, while SsHKT1;1 is involved in maintaining $\mathrm{K}^{+}$and $S s C A X 1$ is involved in maintaining $\mathrm{Ca}^{2+}$ homeostasis under salinity in $S$. salsa [34]. Salinity upregulated expression levels in certain genes, such as choline monooxygenase (CMO), betaine aldehyde dehydrogenase $(\mathrm{BADH})$ and catalase (CAT), elevate the activities of superoxide dismutase (SOD), peroxidase (POD), CAT and glutathione peroxidase (GPx) in S. salsa [35]. High salinity causes metabolic responses, such as depleted amino acids, malate, fumarate, choline and phosphocholine, and elevated betaine and allantoin in the shoots, as well as depleted glucose and fructose, and elevated proline, citrate and sucrose in the roots of S. salsa [35]. A series of metabolomics studies have also been conducted in $S$. salsa under heavy metal stress at ambient salt concentrations [36-38]. However, there are few comprehensive studies on the metabolomics of $S$. salsa in controlled saline conditions, such as determining how salinity affects the metabolites in the species. Widely targeted metabolomics based on multiple reaction monitoring (MRM), using multiple ion monitoring (MIM) survey scans to trigger enhanced product ion (EPI) acquisition to identify metabolites. Compared to nontargeted metabolomics, it is a more sensitive and accurate method for detecting metabolites [39]. Therefore, widely targeted metabolites of $S$. salsa under salt stress were investigated in the present study.

\section{Results}

\section{Data quality assessment}

During the analysis, quality control (QC) samples prepared by mixing the sample extracts were inserted in every 10 test samples to monitor the reproducibility of the analysis process. The accuracy and reproducibility of metabolite detection could be determined using the superimposed display analysis of mass spectrometry total ion current (TIC). TIC is the spectrum obtained by continuously summing the intensity of all ions in the mass spectrum at each time point. The multi-substance extracted ion chromatogram (XIC) can be used determine the ion flux spectrum of each extracted substance in the multiple reaction monitoring mode (MRM). The mass spectral peaks for each color represents the different metabolites detected. The peak area represents the relative content of the corresponding substance. The integration and calibration of peaks were performed using the MultiaQuant software (v 3.0.3).

\section{Qualitative and quantitative metabolites}

The qualitative and quantitative mass spectrometry analysis of metabolites in samples was performed on metabolites based on the Kyoto Encyclopedia of Genes and Genomes (KEGG) database, MetWare database (MWDB), and multiple reaction monitoring (MRM). A total of 639 metabolites were detected based on the metabolic analysis of widely targeted metabolites technique, which included 29 amino acids, 60 amino acid derivatives, 15 benzoic acid derivatives, three pyridine derivatives, nine alcohols and polyols, five cholines, eight catechin derivatives, 16 phenolamides, 53 nucleotide and its derivatives, 15 anthocyanins, 52 flavone, 32 flavonol, one flavonolignan, 29 flavone C-glycosides, 18 flavanone, nine isoflavones, 17 quinates and its derivatives, 32 hydroxycinnamoyl derivatives, six tryptamine derivatives, four alkaloids, 19 carbohydrates, two terpenoids, 16 vitamins, 17 coumarins, five nicotinic acid derivatives, eight 
indole derivatives, 63 organic acids, 63 lipids, and 33 other metabolites (Additional file 1: Table S1).

\section{Principal component analysis (PCA)}

The PCA of the quality control and treatments revealed that the variability of each treatment of samples was small. The samples had similar metabolic characteristics, and the test results were stable and reproducible. In addition, the separation trend between treatments was obvious, indicating the significant metabolic differences between salt treatments (Fig. 1a). The metabolism of these three treatments was clearly separated in the first component (PC1), and the effect of salt treatment on the metabolism of $S$. salsa was obvious (Fig. 1b, c and d).

\section{Orthogonal partial least squares-discriminant analysis (OPLS-DA)}

The OPLS-DA decomposes the $\mathrm{X}$ matrix information into $\mathrm{Y}$ correlation and irrelevance by orthogonal signal correction (OSC) and partial least squares discriminant analysis (PLS-DA) [40]. The difference variables were filtered by eliminating the irrelevant differences. Compared with
PCA, PLS-DA can maximize the distinction between treatments, and is more conducive to finding differential metabolites. The $\mathrm{R}^{2} \mathrm{Y}$ and $\mathrm{Q}^{2}$ scores were all greater than 0.99 in the $200 \mathrm{mM} \mathrm{NaCl}$ vs. CK, $500 \mathrm{mM} \mathrm{NaCl}$ vs. CK, and $500 \mathrm{mM} \mathrm{NaCl}$ vs. $200 \mathrm{mM} \mathrm{NaCl}$ (Fig. 2a, b and c), demonstrating that the results of the salt treatment that led to the differential metabolism of $S$. salsa were correct.

The OPLS-DA model was verified using 200 alignment experiments. The horizontal line corresponded to the $\mathrm{R}^{2}$ and $\mathrm{Q}^{2}$ of the original model, while the red and blue dots represented the $\mathrm{R}^{2}$, and $\mathrm{Q}^{2}$, after replacement, respectively. These results show that the model was meaningful, and that the differential metabolites could be screened according to the VIP value analysis in the subsequent analysis (Fig. 2d, e and f).

\section{Hierarchical cluster analysis (HCA) and volcano plot}

The HCA can evaluate differences in the characteristics of salt treatment that lead to metabolite accumulation, and comprises of intra-treatment homogeneity and inter-treatment variability. With the increase in $\mathrm{NaCl}$ concentration, the difference in the expression of
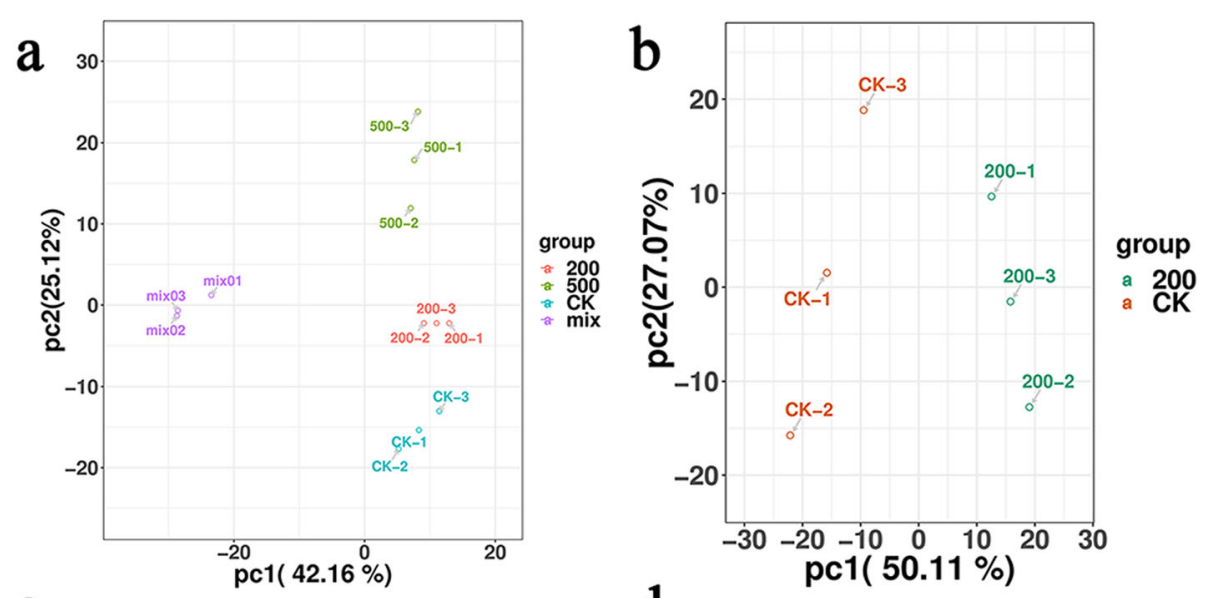

c

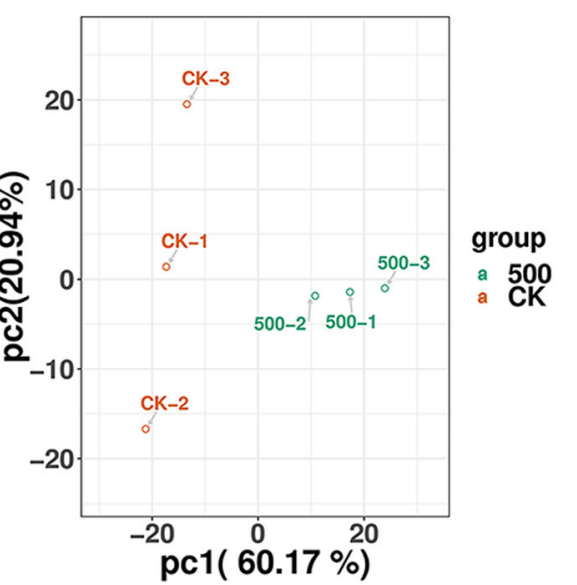

d

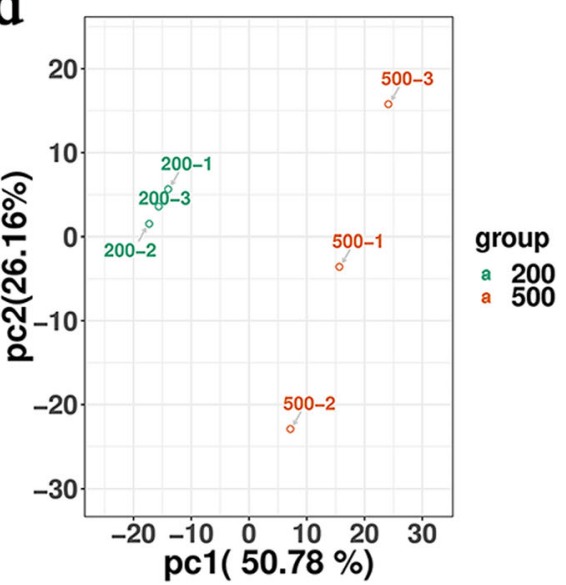

Fig. 1 Principal component analysis (PCA) score map: (a) overall score scatter plot of the PCA model with QC, (b) PCA score map of the $200 \mathrm{mM}$ $\mathrm{NaCl}$ vs. CK (0 mM NaCl), (c) $500 \mathrm{mM} \mathrm{NaCl}$ vs. CK, and (d) $500 \mathrm{mM} \mathrm{NaCl}$ vs. $200 \mathrm{mM} \mathrm{NaCl}$ 

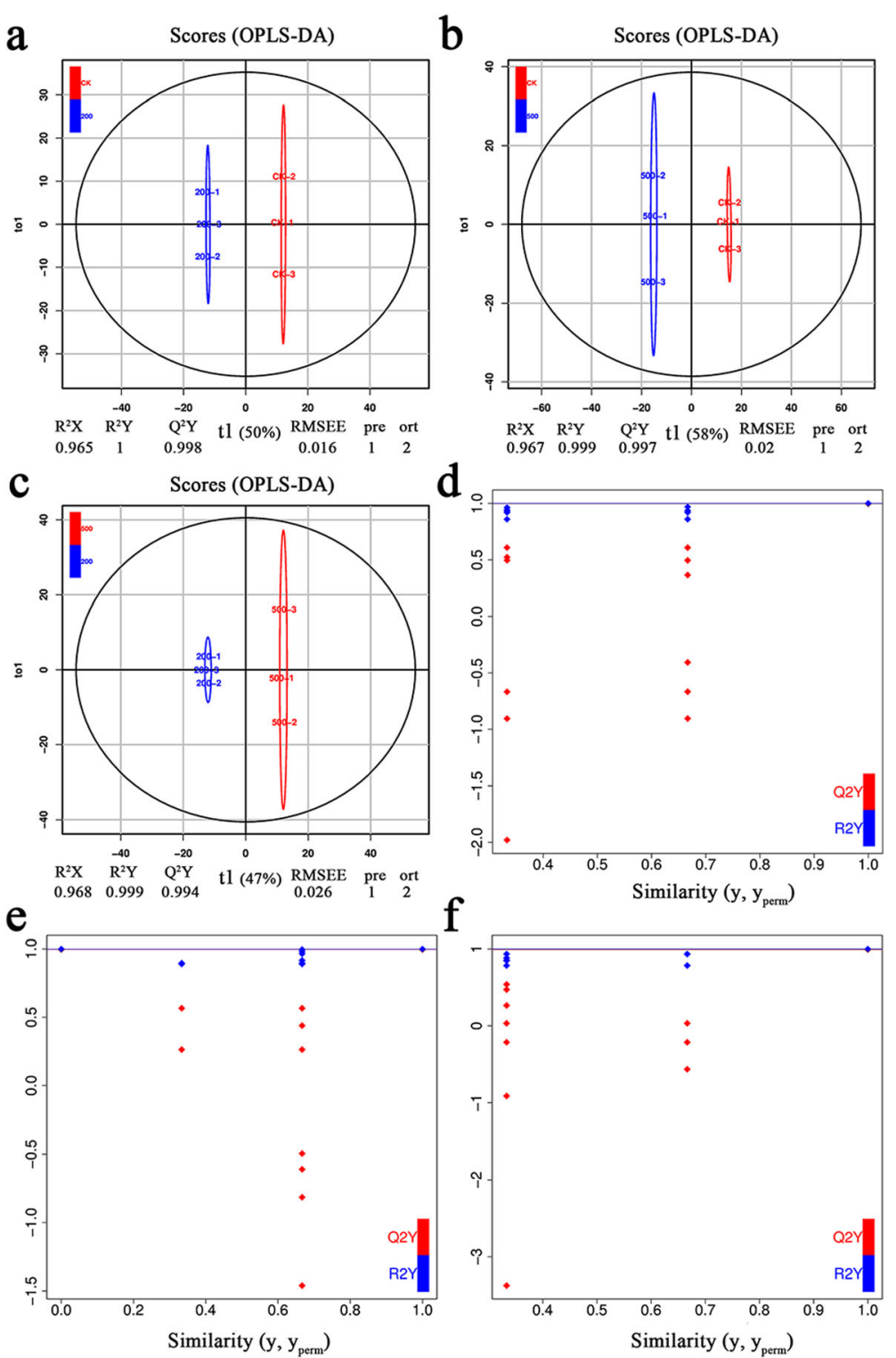

Fig. 2 OPLS-DA scores and permutation verification: scores of the OPLS-DA model with (a) $200 \mathrm{mM} \mathrm{NaCl}$ vs. CK (0 mM NaCl), (b) $500 \mathrm{mM} \mathrm{NaCl}$ vs. CK, and (c) $500 \mathrm{mM} \mathrm{NaCl}$ vs. $200 \mathrm{mM} \mathrm{NaCl}$; OPLS-DA permutation analysis model verification chart of (d) $200 \mathrm{mM} \mathrm{NaCl}$ vs. CK, (e) $500 \mathrm{mM} \mathrm{NaCl} \mathrm{vs.} \mathrm{CK,} \mathrm{and} \mathrm{(f)} 500$ $\mathrm{mM} \mathrm{NaCl}$ vs. $200 \mathrm{mM} \mathrm{NaCl}$. $\mathrm{R}^{2} Y$ and $\mathrm{Q}^{2}$ represent the interpretation rate of the model to the $Y$ matrix and the prediction ability of the model, respectively. A value closer to 1 means that the model is more stable and reliable, and when $Q^{2}$ is $>0.9$, the model is excellent. The horizontal line corresponds to the $R^{2}$ and $Q^{2}$ of the original model, and the red and blue points represent the $R^{21}$ and $Q^{2 t}$ of the model after $Y$ replacement, respectively

metabolites increased (Fig. 3a). The points in the volcano map represents the metabolites, and the abscissa and ordinate represents the logarithm and VIP values of the quantitative difference of metabolites in the two samples, respectively. Metabolites with a fold change of $\geq 2$, a fold change of $\leq 0.5$, and a VIP of $\geq 1$ were selected. The metabolites screened under the above conditions had significant differences. There was no difference in the expression of most metabolites, and the number of increases in differentially expressed metabolites was close to the number of decreases (Fig. 3b, c and d).

\section{Statistical analysis of differential metabolites}

There were 253 metabolites with different content in all 639 metabolites (Additional file 1: Table S1). These were divided into 10 categories: amino acid and its derivatives, phenolamides and phenolic acids, nucleotide and its derivates, flavonoids, lipids, carbohydrates, vitamins, indole derivatives, organic acids, and others (Table 1). Compared to controls, there were 101 differential metabolites in the $200 \mathrm{mM} \mathrm{NaCl}$ treatment, in which 61 metabolites were decreased, while 40 metabolites were increased. Furthermore, there were 199 differential metabolites in 

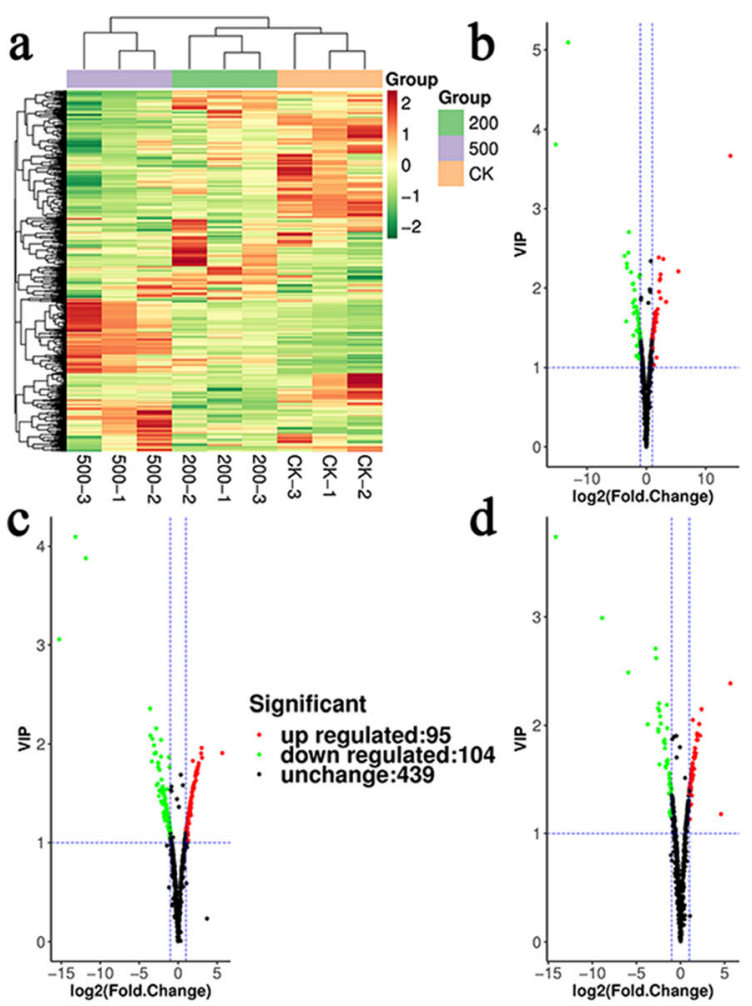

\section{Significant}

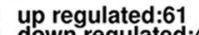

down regulated:46

unchange:530

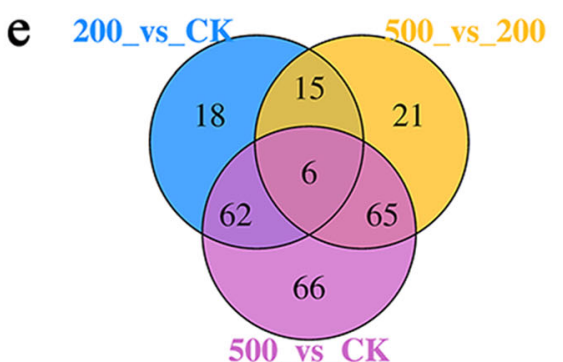

Fig. 3 Hierarchical cluster analysis (HCA), and volcano plot and venn diagram: (a) the heat map shows the differential expression of metabolites between $\mathrm{CK}(0 \mathrm{mM} \mathrm{NaCl}), 200$ and $500 \mathrm{mM} \mathrm{NaCl}$. The green color indicates the decrease of differentially expressed metabolites, while the red color indicates the increase of differentially expressed metabolites; the volcano plot: (b) $200 \mathrm{mM} \mathrm{NaCl}$ vs. CK, (c) $500 \mathrm{mM} \mathrm{NaCl}$ vs. CK, and (d) 500 $\mathrm{mM} \mathrm{NaCl}$ vs. $200 \mathrm{mM} \mathrm{NaCl}$. The green dots in the figure represent the differentially expressed metabolites that were decreased, the red dots represent the increased differentially expressed metabolites, and the black color indicates the detected metabolites, but there was no significant difference; (e) the Venn diagram shows overlapping and specific differential metabolites from CK, 200 and $500 \mathrm{mM} \mathrm{NaCl}$

the $500 \mathrm{mM} \mathrm{NaCl}$ treatment, in which 104 metabolites were decreased, while 95 metabolites were increased. In addition, there were 107 differential metabolites in the $500 \mathrm{mM} \mathrm{NaCl}$ treatment, when compared to the 200 $\mathrm{mM} \mathrm{NaCl}$ treatment, in which 46 metabolites were decreased, while 61 metabolites were increased (Table 1 and Additional file 2: Table S2).

A total of 68 metabolites differed between the low-salt and high-salt treatment, when compared to controls, in which 45 metabolites were decreased, while 22 metabolites were increased. However, 4-(aminomethyl)-5-(hydroxymethyl)-2-methylpyridin-3-ol and dihydromyricetin were barely detected in the $200 \mathrm{mM}$ and $500 \mathrm{mM} \mathrm{NaCl}$ treatments. The most decreased substances were tangeretin, L-ascorbate, 3-hydroxy-3-methylpentane-1, and 5-dioic acid ( $\operatorname{LogFC}<-2.5)$, while the most increased substance was 2' -hydroxygenistein (LogFC $>2.8$ ). Moreover, imidazole-4-acetate was increased after the $200 \mathrm{mM} \mathrm{NaCl}$ treatment, but was decreased after the $500 \mathrm{mM} \mathrm{NaCl}$ treatment (Additional file 3: Table S3).

\section{Functional annotation and enrichment analysis of differential metabolites}

The results of these differentially significant metabolite annotations were classified according to the type of pathway in the KEGG database (http://www.genome.jp/kegg/). 
Table 1 Numbers of differential metabolites in the leaves of $S$. salsa. Plants were cultured at 0, 200 and $500 \mathrm{mM} \mathrm{NaCl}$ for two days

\begin{tabular}{|c|c|c|c|c|c|c|}
\hline \multirow[t]{2}{*}{ Group name Class } & \multicolumn{2}{|c|}{200 vs 0} & \multicolumn{2}{|c|}{500 vs 200} & \multicolumn{2}{|c|}{500 vs 0} \\
\hline & $\overline{U p}$ & Down & Up & Down & $\overline{U p}$ & Down \\
\hline Amino acid and its derivatives & 1 & 13 & 14 & 3 & 13 & 14 \\
\hline Phenolamides and phenolic acids & 6 & 8 & 4 & 7 & 5 & 13 \\
\hline Nucleotide and its derivates & 5 & 2 & 11 & 8 & 18 & 11 \\
\hline flavonoids & 9 & 18 & 5 & 14 & 14 & 31 \\
\hline Lipids & 4 & 2 & 19 & 2 & 25 & 3 \\
\hline Others & 3 & 6 & 4 & 6 & 6 & 13 \\
\hline Carbohydrates & 2 & 1 & & 1 & 2 & 3 \\
\hline Vitamins & 1 & 2 & & 2 & 2 & 6 \\
\hline Indole derivatives & 1 & 1 & 3 & 1 & 1 & 2 \\
\hline Organic acids & 8 & 8 & 1 & 2 & 9 & 8 \\
\hline Sig diff & 40 & 61 & 61 & 46 & 95 & 104 \\
\hline All sig diff & 101 & & 107 & & 199 & \\
\hline
\end{tabular}

Differential metabolites were mainly involved in the metabolic pathways and biosynthesis of secondary metabolites, such as flavonoids, phenols and phenolic acids, amino acids and their derivatives, lipids, organic acids, and other small molecules (Fig. 4a, b and c, Additional file 4: Table S4). The changes in content of these metabolites may play an important role in cell membrane structure protection, maintaining cell osmotic potential and resisting the destruction of reactive oxygen species (ROS) (Fig. 5).

\section{Discussion}

Seeds of $S$. salsa contain high oil, and are rich in unsaturated fatty acids, especially linoleic acid. Hence, this can be used as a source of high-quality edible oil [32, 33]. Fatty acid methyl esters extracted from seedlings and seeds, especially 9, 12-octadecandienoic acid methyl ester and $\gamma$ linolenic acid methyl ester, can inhibit the formation of inflammatory factors, and have obvious anti-inflammatory effects [34]. Furthermore, S. salsa can enrich the heavy metals in the soil, such as $\mathrm{Cu}, \mathrm{Zn}, \mathrm{Pb}$ and $\mathrm{As}$, in order to restore contaminated land, and has high value as the modal for understanding plant salt tolerance [34]. However, the comprehensive metabolic response of $S$. salsa under saline conditions has not been investigated.

Euhalophytes decrease their water potential by accumulating organic substances with small molecules and inorganic ions to resist external osmotic stress [34, 41-44]. Soluble sugars and organic acids play important roles in decreasing water potential for plants under osmotic stress [45]. In the present study, salinity induced the increase of certain kinds of soluble sugars and organic acids, when compared to controls (Table 1). Under osmotic stress, the study conducted by Cao et al. (2004) revealed a significant increase in the content of certain free amino acids, including proline (Pro), aspartic acid (Asp), phenylalanine (Phe) and alanine (Ala), in maize [46]. In the present study, high salinity $(500 \mathrm{mM})$ induced the increase of amino acids, when compared to low salinity $(200 \mathrm{mM})$ (Table 1$)$. This means that $S$. salsa under high salinity can accumulate more amino acids, as well as soluble sugars and organic acids, which can decrease the osmotic potential, and help the species deal with osmotic stress. Furthermore, these amino acids can also increase the nutritional value in species such as vegetables. It has been shown that 3methylcrotonyl glycine is an acyl glycine that has been studied in animals, but this is not been reported in plants [47]. Interestingly, 3-methylcrotonyl glycine was detected in S. salsa at $200 \mathrm{mM} \mathrm{NaCl}$, but was barely detected in controls and at $500 \mathrm{mM} \mathrm{NaCl}$ (Additional file 1: Table S1). Hence, the physiological role and molecular mechanism for the metabolism of the substance in S. salsa can be further investigated.

Lipids mediate some important mechanisms to deal with salt stress, such as the process of participating in cell metabolism and maintaining the stability of the cytoskeleton [48-51]. In the present study, $500 \mathrm{mM}$ $\mathrm{NaCl}$ induced the significant differential expression of lipid metabolites, and more unsaturated fatty acids were increased, when compared to those in controls and at $200 \mathrm{mM} \mathrm{NaCl}$ (Table 1). Monoacylglyceride (MAG), lysophosphatidylcholine (LysoPC), octadecatrienoic acid's derivates, $\alpha$-linolenic acid (ALA) and punicic acid are unsaturated fatty acids that are good for human health, especially ALA [52]. The main role of ALA may be as a precursor to long-chain n-3 PUFAs, such as EPA and DHA [53]. ALA may have a function of preventing heart disease and sudden cardiac death $[54,55]$. The increase of $\alpha$-linolenic acid content plays an important role in regulating intracellular fatty acid unsaturation to resist salt stress [56]. In the present study, $500 \mathrm{mM} \mathrm{NaCl}$ increased the content of $\alpha$-linolenic acid, and the products related to metabolic pathways also significantly increased (Additional file 2: Table S2). Hence, the increased content of ALA may play a role in salt tolerance. Meanwhile, this means that planting S. salsa under high salinity should increase its nutritional value as edible vegetables.

Polyphenols are generally divided into two categories: flavonoids (e.g. flavones, flavanones, flavonols and catechins) and phenolic acids (e.g. hydroxybenzoic acid, hydroxycinnamic acid and quinic acid) [57-59]. Polyphenols have high antioxidant activity, and can prevent cardiovascular diseases and cancers [60, 61]. Therefore, these have been applied in functional foods, cosmetics and medicine [62]. In the present study, different contents of 60 flavonoids and 28 phenolic acids under salt treatment were detected (Additional file 2: Table S2). 


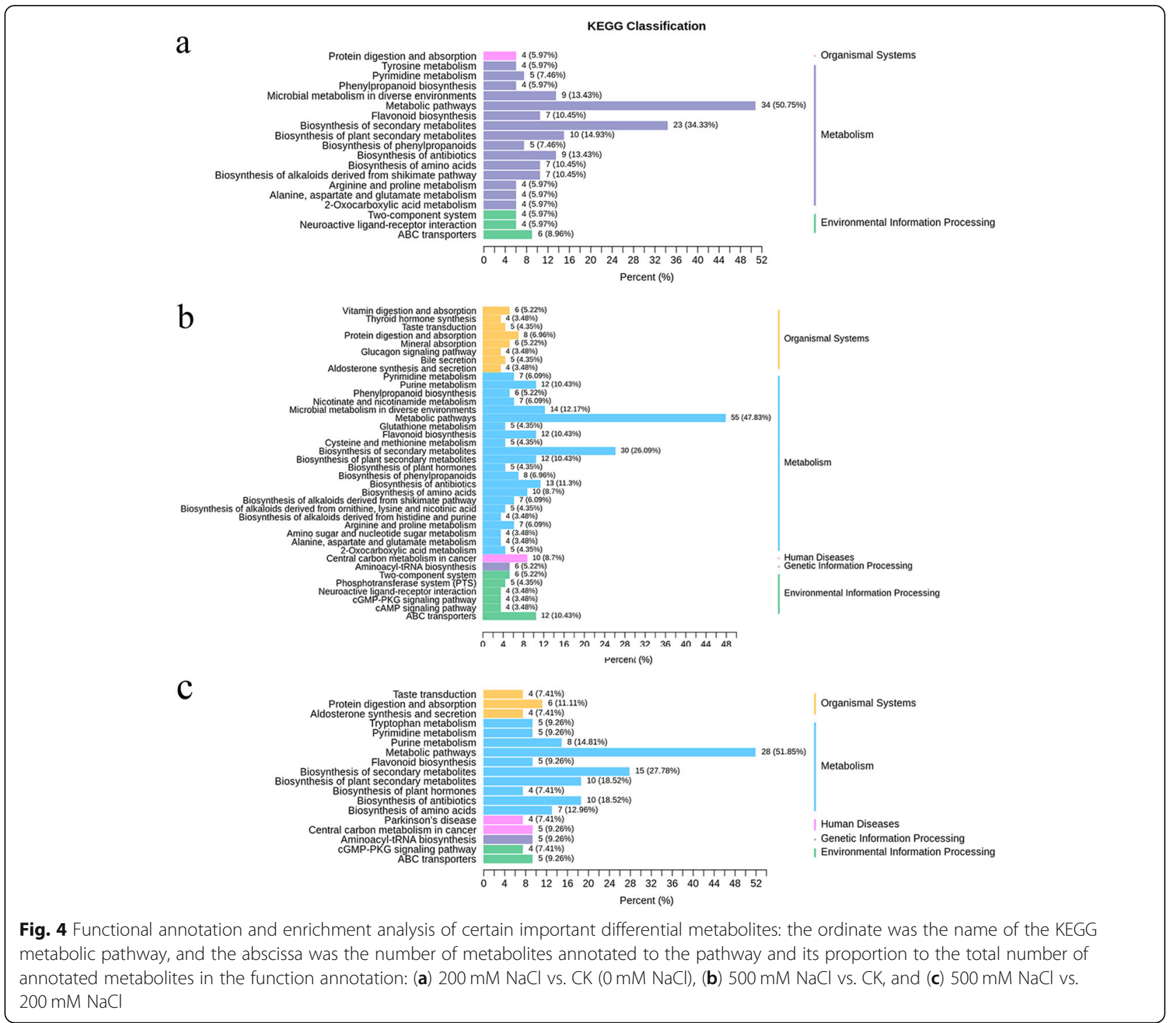

The content of 5-O-p-coumaroylquinic acid, N-acetyl tryptamine, 2'-hydroxygenistein, morin, 2,4-dihydroxybenzoic acid, N', N", N"'-p-coumaroyl-cinnamoyl-caffeoyl spermidine, butin, quercetin, isorhamnetin, homoeriodictyol, hesperetin, naringenin, protocatechuic aldehyde and phloretin significantly increased with salinity. Among these, certain substances have special functions. For example, quercetin is an anti-oxidative flavonoid widely distributed in plants, and is a promising agent for cancer prevention [63-66]. However, more antioxidants, such as flavonoids and polyphenols, were decreased by salinity in the present study (Table 1 ). In S. salsa, the activities of Mn-SOD and several isoforms of Fe-SOD and CuZn-SOD increased under salinity [67]. The overexpression of Ss.SAPX (a gene of the stromal APX in $S$. salsa) increased the salt tolerance of transgenic Arabidopsis plants during both the germination and vegetative growth stages [68]. S. salsa is highly salt tolerant, and grows as well with $400 \mathrm{mM} \mathrm{NaCl}$, when compared to $10 \mathrm{mM} \mathrm{NaCl}$ [69]. The distinctive trait of salt tolerance in euhalophytes, such as $S$. salsa, is to maintain ion homeostasis, including $\mathrm{Na}^{+}$accumulation in the vacuoles [70], $\mathrm{Na}^{+}$and $\mathrm{Cl}^{-}$exclusion through the roots [70, 71], and $\mathrm{K}^{+}[72]$ and $\mathrm{Ca}^{2+}$ [73] homeostasis. Scavenging ROS may be the subsequent mechanism in salt tolerance only when ion homeostasis is destroyed in S. salsa. In addition, S. salsa may scavenge ROS, and mainly relies on enzymatic components, rather than on non-enzymatic components. The role of enzymatic and non-enzymatic components in scavenging ROS should be further investigated. Based on the present results, this study demonstrated that the reconstitution of the metabolic homeostasis of $S$. salsa under salt stress included: 1) the accumulation of primary metabolites (e.g. amino acids, soluble sugars, organic acids, lipids) exerts resistance to 


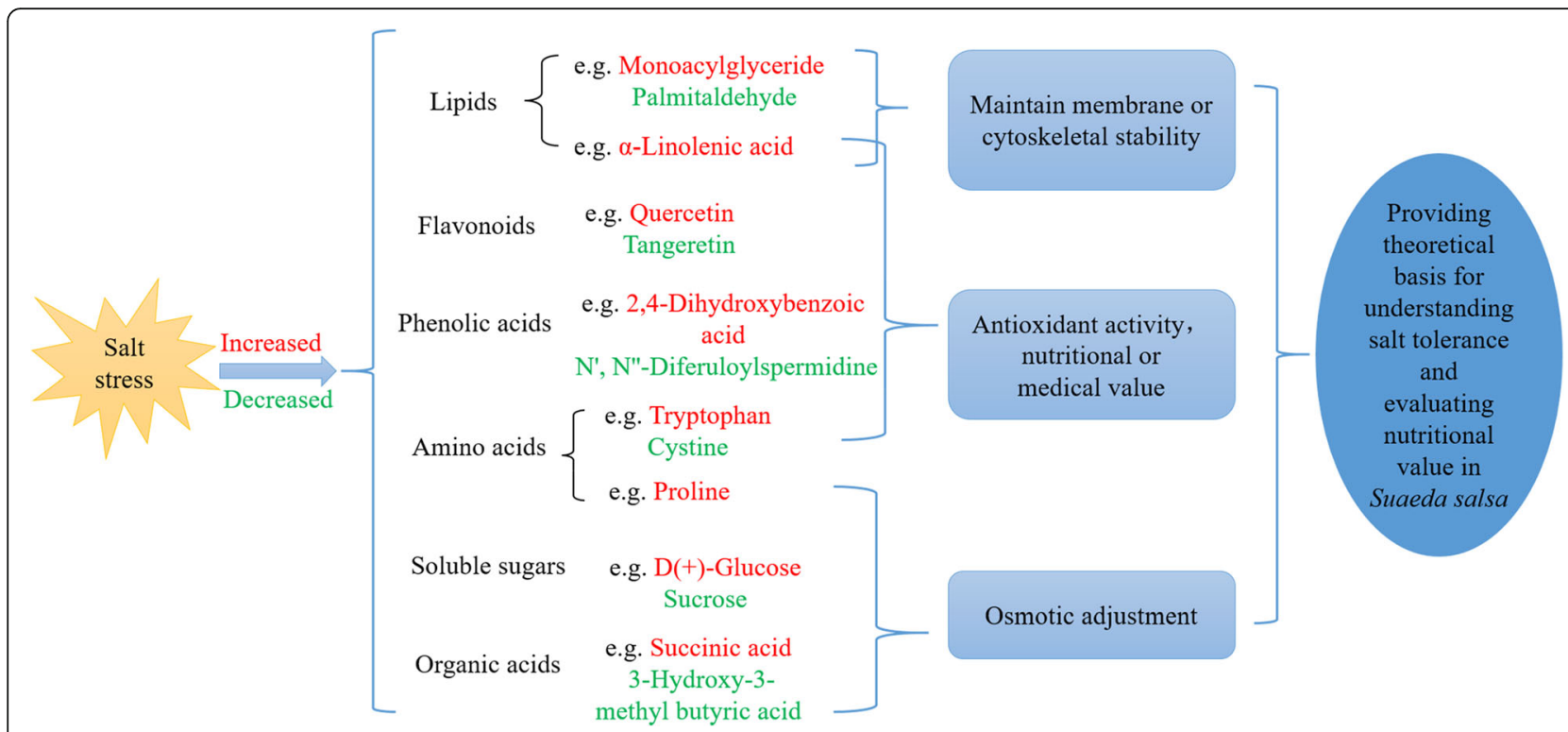

Fig. 5 The outline for metabolic response of S. salsa under salinity stress. The red represents increased, while the green represents decreased

osmotic stress and maintains the osmotic potential and metabolism of cells; 2) secondary metabolites may play an important role as antioxidants and regulatory substances including quercetin, 2,4-dihydroxybenzoic acid, isorhamnetin, 2'-hydroxygenistein, and other metabolites that were significantly inhibited or induced by salt. These metabolites have special nutritional and medicinal value, and also greatly increase the application value of S. salsa (Fig. 6).

\section{Conclusion}

The present study used widely targeted metabolites based on the UPLC-MS/MS detection platform to analyze the metabolic differences of $S$. salsa under different $\mathrm{NaCl}$ concentrations. The present study comprehensively analyzed the metabolic response of $S$. salsa under salt stress from the perspective of omics, providing an important theoretical basis for understanding salt tolerance and evaluating the nutritional value of S. salsa.

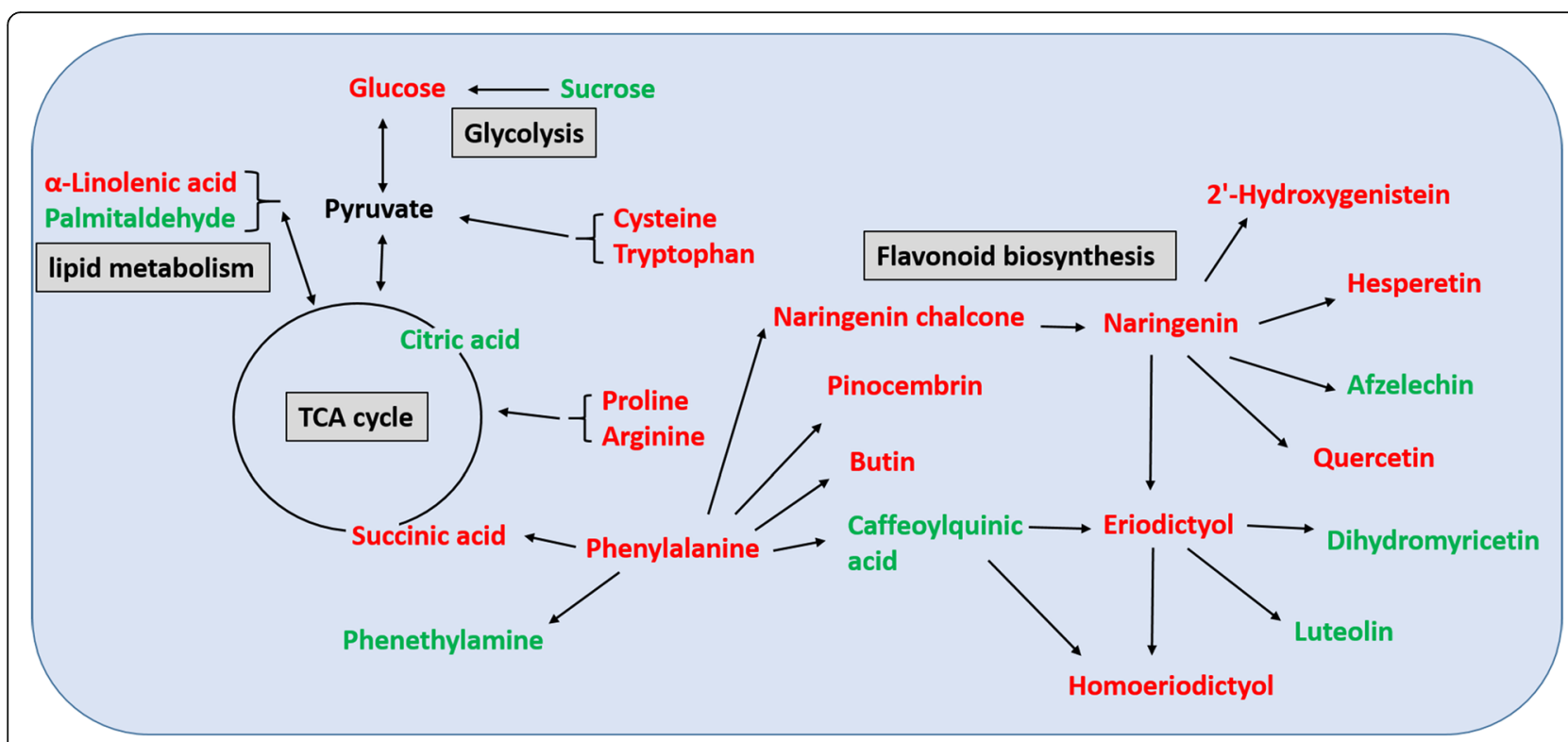

Fig. 6 Schematic presentation of the pathway for certain important metabolites as affected by salinity in S. salsa. The red represents increased, while the green represents decreased 


\section{Methods}

\section{Samples}

The seeds of $S$. salsa were obtained from Dongying Research Academy of Agriculture Science, China. Dry seeds were stored in a fridge at $<4{ }^{\circ} \mathrm{C}$ before use.

In late March 2018, the brown seeds of S. salsa were planted in each plastic pot, which had $2 \mathrm{~kg}$ of rinsed river sand. The seedlings were cultured in a glasshouse under natural light. The temperature was $24 \pm 4{ }^{\circ} \mathrm{C}$ during the day and $18 \pm 4{ }^{\circ} \mathrm{C}$ at night in the glasshouse. The seedlings were watered every day with $1 \mathrm{mM}$ of $\mathrm{NO}_{3}{ }^{-}-\mathrm{N}$ nutrient solution [70]. The $\mathrm{pH}$ of the solution was adjusted to $6.2 \pm 0.1$ with $\mathrm{KOH}$ and $\mathrm{H}_{2} \mathrm{SO}_{4}$.

After the seedlings were pre-cultured for 50 days, 15 seedlings in each pot were left and treated with 0 (as a control, which is indicated as CK in the figures), 200 and $500 \mathrm{mM} \mathrm{NaCl}$ (three pots for each concentration of $\mathrm{NaCl}$ ), which was prepared with $1 \mathrm{mM} \mathrm{NO}_{3}{ }^{-}-\mathrm{N}$ nutrient solution [70]. In order to avoid osmotic shock, $\mathrm{NaCl}$ was added in increments of $50 \mathrm{mM}$ per day. After the highest salinity concentration was obtained for two days, the matured fresh leaves in the same position were harvested and frozen in liquid nitrogen. Then, the samples were stored in dry ice and mailed to MetWare for metabolite testing. For each concentration of $\mathrm{NaCl}$, the matured fresh leaves from each pot were harvested for metabolite testing. That is, three replicates were set for each $\mathrm{NaCl}$ treatment.

\section{Sample extraction process}

The leaves were first freeze-dried and then grounded to powder using a grinder (MM 400; Retsch, Germany) at $30 \mathrm{~Hz}$ for $1.5 \mathrm{~min}$. Then, $0.1 \mathrm{~g}$ of the powder was placed in $1 \mathrm{ml}$ of $70 \%$ aqueous methanol at $4{ }^{\circ} \mathrm{C}$ overnight, and vortexed for three times during the period to increase the extraction efficiency. Next, the extract was centrifuged at 10,000 $\mathrm{g}$ for $10 \mathrm{~min}$, and the supernatant was filtered through a microporous membrane $(0.22-\mu \mathrm{m}$ pore size) before LC-MS/MS analysis.

\section{Analysis of metabolites by ultra performance liquid chromatography and tandem mass spectrometry} The ultra-performance liquid chromatography (UPLC) (Shim-pack UFLC SHIMADZU CBM30A, http://www. shimadzu.com.cn/) and tandem mass spectrometry (MS/ MS) (Applied Biosystems 6500 QTRAP) conditions were as follows: column, waters ACQUITY UPLC HSS T3 C18 $1.8 \mu \mathrm{m}, 2.1 \times 100 \mathrm{~mm}$; mobile phase, the aqueous phase was ultrapure water $(0.04 \%$ acetic acid), while the organic phase was acetonitrile $(0.04 \%$ acetic acid); gradient of water/acetonitrile, $95: 5 \mathrm{~V} / \mathrm{V}$ for zero minutes, 5: $95 \mathrm{~V} / \mathrm{V}$ for $11.0 \mathrm{~min}, 5: 95 \mathrm{~V} / \mathrm{V}$ for $12.0 \mathrm{~min}, 95: 5 \mathrm{~V} / \mathrm{V}$ for $12.1 \mathrm{~min}$, and $95: 5 \mathrm{~V} / \mathrm{V}$ for $15.0 \mathrm{~min}$; flow rate of $0.4 \mathrm{ml} /$ min; column temperature at $40{ }^{\circ} \mathrm{C}$; injection volume at
$2 \mu \mathrm{l}$. The electrospray ionization (ESI) temperature was $500{ }^{\circ} \mathrm{C}$, the mass spectrometry voltage was $5500 \mathrm{~V}$, the curtain gas (CUR) was $25 \mathrm{psi}$, and the collision induced dissociation (CAD) parameter was set as high. In the triple quadrupole (QQQ), each ion pair was scanned for detection based on the optimized decompression potential (DP) and collision energy (CE) [39].

Qualitative and quantitative determination of metabolites Based on the public metabolite database (e.g. MassBank or KNApSAcK) and the self-built database MetWare database (MWDB), the material was qualitative by secondary spectral information, while the isotope signal and the repetitive signal are removed during the analysis.

The metabolites were quantified using multiple reaction monitoring (MRM) of triple quadrupole mass spectrometry. The ions corresponding to other molecular weight substances were excluded, and the precursor ions of the target substance were screened. Meanwhile, in the collision cell, the precursor ions were ionized to break and form fragment ions, and the characteristic fragment ions were selected by triple quadrupole filtration. This makes the quantitative results more accurate and repeatable. Peak area integration was performed on the obtained metabolite mass spectral peaks, and the mass spectral peaks of the metabolites in different samples were integrated [74].

\section{Statistical analysis}

Multivariate statistical analysis methods, including principal component analysis (PCA) and orthogonal partial least squares discriminant analysis (OPLS-DA), were used. PCA was used to recombine original variables into new, mutually independent variables through orthogonal transformations, revealing the internal structure of multiple variables through a few principal components [75]. Based on the results of the OPLS-DA, a multivariate analysis of variable importance in the project (VIP) in the OPLS-DA model could be used to initially screen for metabolites with differences. At the same time, differential metabolites could be further screened by combining the $P$-values or fold changes in the univariate analysis. Finally, the differential metabolites were precisely mined. Hierarchical cluster analysis (HCA) was performed on the accumulation patterns of metabolites between different samples using the $\mathrm{R}$ software (www.r-project.org/). The KEGG database was used to annotate the differential metabolites [76].

\section{Additional files}

Additional file 1: Table S1. All detected metabolites in the leaves of S. salsa treated with different concentrations of $\mathrm{NaCl}$. (XLSX $109 \mathrm{~kb}$ )

Additional file 2: Table S2. All differential metabolites in the leaves of S. salsa treated with different concentrations of $\mathrm{NaCl}$. (XLSX 68 kb) 
Additional file 3: Table S3. Differential metabolites in the leaves of S. salsa at salinity, when compared to controls. (XLSX $30 \mathrm{~kb}$ )

Additional file 4: Table S4. Differentially significant metabolite annotations classified by KEGG pathways. (XLSX 44 kb)

\section{Abbreviations}

MRM: Multiple reaction monitoring; MS/MS: Tandem mass spectrometry; QQQ: Triple quadrupole; UPLC: Ultra performance liquid chromatography; VIP: Variable importance in the project

\section{Acknowledgements}

Not applicable.

\section{Authors' contributions}

QL and JS conceived and designed the experimental plan. QL participated in sample collection and experiments and wrote the manuscript. JS revised the manuscript. Both authors have read and approved the manuscript.

\section{Funding}

The work is supported from National Natural Science Research Foundation of China (U1803233, 31570392 to JS), and State Key Laboratory of Desert and Oasis Ecology, Xinjiang Institute of Ecology and Geography, Chinese Academy of Sciences (G2019-02-04 to JS). The above funding was used for the design of the study and collection, analysis, and interpretation of data in writing the manuscript.

\section{Availability of data and materials}

The datasets used and/or analysed during the current study available from the corresponding author on reasonable request.

\section{Ethics approval and consent to participate}

The research in this manuscript was approved by the Research Leader from Shandong Provincial Key Laboratory of Plant Stress Research and is part of the mission of the National Natural Science Research Foundation of China (No. U1803233, 31570392). All experiments conducted are in compliance with relevant laws in China.

\section{Consent for publication}

Not applicable.

\section{Competing interests}

The authors declare that they have no competing interests.

Received: 10 April 2019 Accepted: 30 August 2019

Published online: 06 September 2019

\section{References}

1. Munns R. Genes and salt tolerance: bringing them together. New Phytol. 2005;167(3):645-63.

2. Shabala S. Learning from halophytes: physiological basis and strategies to improve abiotic stress tolerance in crops. Ann Bot. 2013;112(7):1209-21.

3. Fiehn O, Kopka J, Dörmann P, Altmann T, Trethewey RN, Willmitzer L. Metabolite profiling for plant functional genomics. Nat Biotechnol. 2000; 18(11):1157-61.

4. Fukusaki E, Kobayashi A. Plant metabolomics: potential for practical operation. J Biosci Bioeng. 2005;100(4):347-54.

5. Fiehn $\mathrm{O}$, Kloska S, Altmann T. Integrated studies on plant biology using multiparallel techniques. Curr Opin Biotech. 2001;12(1):82-6.

6. Weckwerth W. Metabolomics in systems biology. Annu Rev Plant Biol. 2003; 54:669-89.

7. Fiehn O. Metabolomics-the link between genotypes and phenotypes. Plant Mol Biol. 2002:48(1-2):155-71.

8. Töpfer N, Kleessen S, Nikoloski Z. Integration of metabolomics data into metabolic networks. Front Plant Sci. 2015;6:49.

9. Dettmer K, Aronov PA, Hammock BD. Mass spectrometry-based metabolomics. Mass Spectrom Rev. 2007;26(1):51-78.

10. Arbona V, Manzi M, Ollas CD, Gómez-Cadenas A. Metabolomics as a tool to investigate abiotic stress tolerance in plants. Int J Mol Sci. 2013;14(3):4885-911.

11. Shulaev V, Cortes D, Miller G, Mittler R. Metabolomics for plant stress response. Physiol Plantarum. 2008;132(2):199-208.
12. Hong J, Yang L, Zhang D, Shi J. Plant metabolomics: an indispensable system biology tool for plant science. Int J Mol Sci. 2016;17(6):767.

13. Obata T, Fernie AR. The use of metabolomics to dissect plant responses to abiotic stresses. Cell Mol Life Sci. 2012;69(19):3225-43.

14. Durek $P$, Walther $D$. The integrated analysis of metabolic and protein interaction networks reveals novel molecular organizing principles. BMC Syst Biol. 2008;2(1):100

15. Sanchez DH, Pieckenstain FL, Szymanski J, Erban A, Bromke M, Hannah MA, Kraemer U, Kopka J, Udvardi MK. Comparative functional genomics of salt stress in related model and cultivated plants identifies and overcomes limitations to translational genomics. PLoS One. 2011;6(2):e17094.

16. D'Auria JC, Gershenzon J. The secondary metabolism of Arabidopsis thaliana: growing like a weed. Curr Opin Plant Biol. 2005;8(3):308-16.

17. Yan N, Du Y, Liu X, Chu MJ, Shi J, Zhang HB, Liu YH, Zhang ZF. A comparative UHPLC-QqQ-MS-based metabolomics approach for evaluating Chinese and north American wild rice. Food Chem. 2019;275:618-27.

18. Kusano M, Fukushima A, Redestig H, Saito K. Metabolomic approaches toward understanding nitrogen metabolism in plants. J Exp Bot. 2011;62(4): 1439-53.

19. Kato H, Izumi Y, Hasunuma T, Matsuda F, Kondo A. Widely targeted metabolic profiling analysis of yeast central metabolites. J Biosci Bioeng. 2012;113(5):665-73.

20. Kim JK, Bamba T, Harada K, Fukusaki E, Kobayashi A. Time-course metabolic profiling in Arabidopsis thaliana cell cultures after salt stress treatment. J Exp Bot. 2007:58(3):415-24.

21. Gavaghan CL, Li JV, Hadfield ST, Hole S, Nicholson JK, Wilson ID, Howe PWA Stanley PD, Holmes E. Application of NMR-based metabolomics to the investigation of salt stress in maize (Zea mays). Phytochem Analysis. 2011; 22(3):214-24.

22. Widodo PJH, Newbigin ED. Tester M, Bacic a, Roessner U. metabolic responses to salt stress of barley (Hordeum vulgare L.) cultivars, Sahara and clipper, which differ in salinity tolerance. J Exp Bot. 2009;60(14):4089-103.

23. Li WQ, Yamaguchi S, Khan MA, An P, Liu XJ, Tran LSP. Roles of gibberellins and abscisic acid in regulating germination of Suaeda salsa dimorphic seeds under salt stress. Front Plant Sci. 2016;6:1235.

24. Song J, Shi WW, Liu RR, Xu YG, Sui N, Zhou JC, Feng G. The role of the seed coat in adaptation of dimorphic seeds of the euhalophyte Suaeda salsa to salinity. Plant Spec Biol. 2017;32(2):107-14.

25. Xu YG, Liu RR, Sui N, Shi WW, Wang L, Tian CY, Song J. Changes in endogenous hormones and seed coat phenolics during seed storage of two Suaeda salsa populations. Aust J Bot. 2016;64(4):325-32.

26. Zhou JC, Fu TT, Sui N, Guo JR, Feng G, Fan JL, Song J. The role of salinity in seed maturation of the euhalophyte Suaeda salsa. Plant Biosyst. 2016;150(1):83-90.

27. Chen TS, Yuan F, Song J, Wang BS. Nitric oxide participates in waterlogging tolerance through enhanced adventitious root formation in the euhalophyte Suaeda salsa. Funct Plant Biol. 2016;43(3):244-53.

28. Guo JR, Suo SS, Wang BS. Sodium chloride improves seed vigour of the euhalophyte Suaeda salsa. Seed Sci Res. 2015;25(3):335-44.

29. Guo JR, Li YD, Han GL, Song J, Wang BS. NaCl markedly improved the reproductive capacity of the euhalophyte Suaeda salsa. Funct Plant Biol. 2018;45(3):350-61.

30. Wang FX, Xu YG, Wang S, Shi WW, Liu RR, Feng G, Song J. Salinity affects production and salt tolerance of dimorphic seeds of Suaeda salsa. Plant Physiol Bioch. 2015;95:41-8.

31. Wang FX, Yin CH, Song YP, Li Q, Tian CY, Song J. Reproductive allocation and fruit-set pattern in the euhalophyte Suaeda salsa in controlled and field conditions. Plant Biosyst. 2018a;152(4):749-58.

32. Zhao YQ, Ma YC, Duan HM, Liu RR, Song J. Traits of fatty acid accumulation in dimorphic seeds of the euhalophyte Suaeda salsa in saline conditions. Plant Biosyst. 2019;153(4):514-20.

33. Zhao YQ, Ma YC, Li Q, Yang Y, Guo JR, Song J. Utilization of stored lipids during germination in dimorphic seeds of euhalophyte Suaeda salsa. Funct Plant Biol. 2018a;45(10):1009-16.

34. Song J, Wang BS. Using euhalophytes to understand salt tolerance and to develop saline agriculture: Suaeda salsa as a promising model. Ann Bot. 2015;115(3):541-53.

35. Wu HF, Liu XL, You LP, Zhang LB, Zhou D, Feng JH, Zhao JM, Yu JB. Effects of salinity on metabolic profiles, gene expressions, and antioxidant enzymes in halophyte Suaeda salsa. J Plant Growth Regul. 2012;31(3):332-41.

36. Liu XL, Yang CY, Zhang LB, Li LZ, Liu SJ, Yu JB, Liu LP, Zhou D, Xia CH, Zhao JM, Wu HF. Metabolic profiling of cadmium-induced effects in one pioneer 
intertidal halophyte Suaeda salsa by NMR-based metabolomics. Ecotoxicology. 2011;20(6):1422-31.

37. Wu H, Liu X, Zhao J, Yu J, Pang Q, Feng J. Toxicological effects of environmentally relevant lead and zinc in halophyte Suaeda salsa by NMRbased metabolomics. Ecotoxicology. 2012;21(8):2363-71.

38. Liu X, Wu H, Ji C, Wei L, Zhao J, Yu J. An integrated proteomic and metabolomic study on the chronic effects of mercury in Suaeda salsa under an environmentally relevant salinity. PLoS One. 2013;8(5):e64041.

39. Chen W, Gong L, Guo Z, Wang WS, Zhang HY, Liu XQ, Yu SB, Xiong LH, Luo J. A novel integrated method for large-scale detection, identification, and quantification of widely targeted metabolites: application in the study of rice metabolomics. Mol Plant. 2013;6(6):1769-80.

40. Bylesjö M, Rantalainen M, Cloarec O, Nicholson JK, Holmes E, Trygg J. OPLS discriminant analysis: combining the strengths of PLS-DA and SIMCA classification. J Chemom. 2006;20(8-10):341-51.

41. Flowers TJ, Colmer TD. Salinity tolerance in halophytes. New Phytol. 2008; 179(4):945-63.

42. Li X, Liu Y, Chen M, Song YP, Song J, Wang BS, Feng G. Relationships between ion and chlorophyll accumulation in seeds and adaptation to saline environments in Suaeda salsa populations. Plant Biosyst. 2012a;146:142-9.

43. Munns R, Tester M. Mechanisms of salinity tolerance. Annu Rev Plant Biol. 2008:59:651-81.

44. Song J, Zhou JC, Zhao WW, Xu H, Wang FX, Xu YG, Tian CY. Effects of salinity and nitrate on production and germination of dimorphic seeds applied both through the mother plant and exogenously during germination in Suaeda salsa. Plant Spec Biol. 2016;31 (1):19-28.

45. Song J, Feng G, Tian CY, Zhang FS. Osmotic adjustment traits of Suaeda physophora Haloxylon ammodendron and Haloxylon persicum in field or controlled conditions. Plant Sci. 2006;170(1):113-9.

46. Cao R, Liang Z, Wu Y, Zhang L, Kang S. The change of amino acids in leaves of maize seedlings under alternative split--root osmotic stress. Agric Res Arid Areas (in Chinese with English abstract). 2004;22(1):49-53.

47. Moura AP, Ribeiro CAJ, Zanatta Â, Busanello ENB, Tonin AM, Wajner M. 3Methylcrotonylglycine disrupts mitochondrial energy homeostasis and inhibits synaptic $\mathrm{Na}^{+}, \mathrm{K}^{+}$-ATPase activity in brain of young rats. Cell Mol Neurobiol. 2012;32(2):297-307.

48. Hou Q, Ufer G, Bartels D. Lipid signalling in plant responses to abiotic stress. Plant Cell Environ. 2016;39(5):1029-48.

49. Sui N, Li M, Li K, Song J, Wang BS. Increase in unsaturated fatty acids in membrane lipids of Suaeda salsa $\mathrm{L}$. enhances protection of photosystem II under high salinity. Photosynthetica. 2010;48(4):623-9.

50. Sui N, Han GL. Salt-induced photoinhibition of PSII is alleviated in halophyte Thellungiella halophila by increases of unsaturated fatty acids in membrane lipids. Acta Physiol Plant. 2014;36(4):983-92.

51. Sui N, Tian SS, Wang WQ, Wang MJ, Fan H. Overexpression of glycerol-3phosphate acyltransferase from Suaeda salsa improves salt tolerance in Arabidopsis. Front Plant Sci. 2017;8:1337.

52. Zhao YQ, Yang Y, Song YP, Li Q, Song J. Analysis of storage compounds and inorganic ions in dimorphic seeds of euhalophyte Suaeda salsa. Plant Physiol Bioch. 2018b;130:511-6.

53. Blanchard H, Pédrono F, Boulier-Monthéan N, Catheline D, Rioux V, Legrand P. Comparative effects of well-balanced diets enriched in a-linolenic or linoleic acids on LC-PUFA metabolism in rat tissues. Prostag Leukotr Ess. 2013;88(5):383-9.

54. Hu FB, Stampfer MJ, Manson JE, Rimm EB, Wolk A, Colditz GA, Hennekens $\mathrm{CH}$, Willett WC. Dietary intake of a-linolenic acid and risk of fatal ischemic heart disease among women. Am J Clin Nutr. 1999;69(5):890-7.

55. Sinclair AJ, Attar-Bashi NM, Li D. What is the role of a-linolenic acid for mammals? Lipids. 2002;37(12):1113-23.

56. Jiang $X L$, Wei $D$. Analysis of lipid in Chlamydomonas nivalis to salt stress by GC/MS. Sci Technol Food Ind (in Chinese with English abstract). 2010;31(7):71.

57. Li H, Wang X, Li Y, Li P, Wang H. Polyphenolic compounds and antioxidant properties of selected China wines. Food Chem. 2009;112(2):454-60.

58. Robbins RJ. Phenolic acids in foods: an overview of analytical methodology. J Agr Food Chem. 2003;51(10):2866-87.

59. Wang A, Li R, Ren L, Gao XL, Zhang YG, Ma ZM, Ma DF, Luo YH. A comparative metabolomics study of flavonoids in sweet potato with different flesh colors (Ipomoea batatas (L.) lam). Food Chem. 2018b;260:124-34.

60. Ding HR, Hong L, Yang ZQ, Wang M, Wang K, Zhu XM. Progress of study on halophyte Suaeda salsa. Acta Agric Jiangxi (in Chinese with English abstract). 2008;20(8):35-7.
61. Oueslati S, Trabelsi N, Boulaaba M, Legault J, Abdelly C, Ksouri R. Evaluation of antioxidant activities of the edible and medicinal Suaeda species and related phenolic compounds. Ind Crop Prod. 2012;36(1):513-8.

62. Maisuthisakul P, Suttajit M, Pongsawatmanit R. Assessment of phenolic content and free radical-scavenging capacity of some Thai indigenous plants. Food Chem. 2007;100(4):1409-18.

63. Murakami A, Ashida H, Terao J. Multitargeted cancer prevention by quercetin. Cancer Lett. 2008;269(2):315-25.

64. Qiu Y, Liu Q, Beta T. Antioxidant properties of commercial wild rice and analysis of soluble and insoluble phenolic acids. Food Chem. 2010;121(1): 140-7.

65. Rice-Evans CA, Miller NJ, Paganga G. Structure-antioxidant activity relationships of flavonoids and phenolic acids. Free Radical Bio Med. 1996; 20(7):933-56.

66. Sroka Z, Cisowski W. Hydrogen peroxide scavenging antioxidant and antiradical activity of some phenolic acids. Food Chem Toxicol. 2003;41 (6):753-8.

67. Wang $B$, Lüttge $U$, Ratajczak R. Specific regulation of SOD isoforms by $\mathrm{NaCl}$ and osmotic stress in leaves of the C3 halophyte Suaeda salsa L. J Plant Physiol. 2004;161(3):285-93.

68. Li K, Pang CH, Ding F, Sui N, Feng ZT, Wang BS. Overexpression of Suaeda salsa stroma ascorbate peroxidase in Arabidopsis chloroplasts enhances salt tolerance of plants. S Afr J Bot. 2012b;78:235-45.

69. Song J, Chen M, Feng G, Jia YH, Wang BS, Zhang FS. Effect of salinity on growth ion accumulation and the roles of ions in osmotic adjustment of two populations of Suaeda salsa. Plant Soil. 2009;314(1-2):133-41.

70. Liu QQ, Liu RR, Ma YC, Song J. Physiological and molecular evidence for $\mathrm{Na}^{+}$and $\mathrm{Cl}^{-}$exclusion in the roots of two Suaeda salsa populations. Aquat Bot. 2018;146:1-7.

71. Song J, Shi GW, Gao B, Fan H, Wang BS. Waterlogging and salinity effects on two Suaeda salsa populations. Physiol Plantarum. 2011;141(4):343-51.

72. Shao Q, Han N, Ding T, Zhou F, Wang BS. SsHKT1; 1 is a potassium transporter of the C3 halophyte Suaeda salsa that is involved in salt tolerance. Funct Plant Biol. 2014;41(8):790-802.

73. Han $\mathrm{N}$, Shao $\mathrm{Q}, \mathrm{Bao} \mathrm{H}$, Wang BS. Cloning and characterization of a $\mathrm{Ca}^{2+} / \mathrm{H}^{+}$ antiporter from halophyte Suaeda salsa L. Plant Mol Biol Rep. 2011;29(2): 449-57.

74. Fraga CG, Clowers BH, Moore RJ, Zink EM. Signature-discovery approach for sample matching of a nerve-agent precursor using liquid chromatographymass spectrometry XCMS and Chemometrics. Anal Chem. 2010;82(10):4165-73.

75. Eriksson L, Andersson PL, Johansson E, Tysklind M. Megavariate analysis of environmental QSAR data. Part I-A basic framework founded on principal component analysis (PCA) partial least squares (PLS) and statistical molecular design (SMD). Mol Divers. 2006;10(2):169-86.

76. Kanehisa M, Goto S. KEGG: Kyoto encyclopedia of genes and genomes. Nucleic Acids Res. 2000;28(1):27-30.

\section{Publisher's Note}

Springer Nature remains neutral with regard to jurisdictional claims in published maps and institutional affiliations.

Ready to submit your research? Choose BMC and benefit from:

- fast, convenient online submission

- thorough peer review by experienced researchers in your field

- rapid publication on acceptance

- support for research data, including large and complex data types

- gold Open Access which fosters wider collaboration and increased citations

- maximum visibility for your research: over $100 \mathrm{M}$ website views per year

At $\mathrm{BMC}$, research is always in progress.

Learn more biomedcentral.com/submissions 